\title{
Atenção à saúde dos homens no âmbito da Estratégia Saúde da Família
}

\author{
Men's health care in the scope of the Family Health Strategy
}

Erly Catarina de Moura ${ }^{1}$

Wallace dos Santos ${ }^{1}$

Alice Cristina Medeiros das Neves ${ }^{1}$

Romeu Gomes ${ }^{2}$

Eduardo Schwarz ${ }^{1}$

${ }^{1}$ Ministério da Saúde. Asa Sul. 70.070-923 Brasília DF Brasil.erlycm@usp.br ${ }^{2}$ Instituto Fernandes Figueira, Fiocruz.

\begin{abstract}
The National Policy of Comprehensive Care for Men's Health created the guidelines for the strategies and actions based on comprehensive care, seeking the promotion of health and the prevention of disease duly focused as core issues of the Family Health Strategy (FHS). This article describes the specificities of men's health care in the context of the FHS from the standpoint of the manager, the demands of the men linked to the health units assessed and the practices adopted by the teams. Men's health care was evaluated by interviews with 43 FHS team managers (FHST), systematically selected considering the geographical region, city size and FHS coverage; and by interviewing 86 adult men of the respective FHS coverage area. It was seen that the strategy of the FHST is to address the health-disease process in the family and environmental context. However, in men's health there are still several gaps, from the adaptation of the structure of primary health care through to the motivation and development of actions against the most common health problems of this population group. This situation sometimes limits men's access to health services thereby negating the goal of the Policy.
\end{abstract}

Key words Men's health, Health policies, Gender and health, Family Health Program
Resumo A Politica Nacional de Atenção Integral à Saúde do Homem orientou a formulação de estratégias e ações fundamentando-se na atenção integral, com vistas à promoção da saúde e à prevenção de agravos, tendo sido focalizadas como eixos fundamentais na Estratégia Saúde da Família (ESF). Este artigo teve por objetivo descrever as especificidades da atenção à saúde dos homens no âmbito da ESF, conforme a visão do gestor, a demanda dos homens adstritos às unidades avaliadas e as práticas desenvolvidas pelas equipes. A atenção à saúde do homem foi avaliada por meio de entrevista a 43 gestores de equipes da ESF (EqSF), sorteadas de forma sistemática contemplando as diferentes regiões, porte dos municípios e cobertura da ESF; e pela entrevista de 86 homens adultos, das respectivas áreas de cobertura da ESF. Observou-se que as EqSF têm como estratégia abordar o processo saúde/doença no contexto familiar e ambiental, no entanto, no que se refere à saúde do homem, ainda há lacunas, desde a adequação da estrutura para o atendimento na atenção básica à motivação e desenvolvimento de ações de promoção contra os agravos mais frequentes nesta população, o que, por vezes, têm dificultado o acesso à saúde, por parte da população masculina, distanciando o alcance do objetivo da Política.

Palavras-chave Saúde do homem, Políticas de saúde, Gênero e saúde, Programa Saúde da Família 


\section{Introdução}

Tradicionalmente, os homens não têm suas especificidades reconhecidas e não fazem parte das populações usualmente mais assistidas nos serviços de atenção básica à saúde (ABS). O uso dos serviços de saúde pelos homens difere daquele feito pelas mulheres, concentrando-se na assistência a agravos e doenças, em que a busca por atendimento, em geral, acontece em situações extremas de emergência e/ou em nível especializado ou de urgência. Essa discussão pode ser mais ampliada a partir da perspectiva de gênero, aqui entendida como referência sócio-histórica que, baseada nas diferenças entre os sexos, ordena material e simbolicamente a vida social de homens e mulheres ${ }^{1}$. Nas construções dos modelos de gênero, costuma-se representar o masculino em oposição ao universo feminino. Reflexos dessa oposição têm sido observados em relação aos cuidados em saúde. Nesse sentido, observa-se que homens costumam ter mais dificuldades na busca por assistência em saúde quando veem as necessidades de cuidado como uma tarefa feminina $^{2}$. Assim, eles costumam só chegar aos serviços de saúde com intercorrências graves ou quando se veem impossibilitados de exercer seu papel de trabalhador, ressaltando também que, segundo a visão dos profissionais de saúde, os homens não buscam os serviços para fins preventivos ${ }^{3,4}$.

Dados do Sistema de Informações Ambulatoriais do SUS (SIA/SUS) apontaram que, em 2010, o número total de consultas médicas para homens entre 20 e 59 anos de idade apresentado ao gestor do SUS e registrado no sistema foi de 3.217.197, o que resulta numa média de 0,06 consulta/homem/ano ${ }^{5}$. Esta situação se configura como consequência da histórica priorização da população feminina e infantil pelo próprio setor saúde e também pela dificuldade de verbalização das necessidades de saúde, do próprio homem, no contexto da assistência, que se encontra enraizada à própria noção de invulnerabilidade $e^{6-8}$. Por consequência, ao se sentir invulneráveis, os homens, de maneira geral e em sua pluralidade, se expõem mais a riscos e acabam sendo de fato vulneráveis ${ }^{7}$. Há estudos que também consideram que os homens estão mais vulneráveis aos problemas de saúde, em virtude da baixa acessibilidade aos serviços de atenção primária9. Em face a esta realidade, a Política Nacional de Atenção Integral à Saúde do Homem (PNAISH), instituída em 27 de agosto de 2009, por meio da Portaria GM/MS no 1944, orientou a formulação de diretrizes e ações voltadas fundamentalmente para a atenção integral, com vistas à prevenção e promoção da saúde, à qualidade de vida e à educação, como dispositivos estratégicos de incentivo às mudanças comportamentais ${ }^{10}$. Adicionalmente, a promoção da saúde e a prevenção de agravos têm sido focalizadas como eixos prioritários na Estratégia Saúde da Família (ESF) ${ }^{11}$.

A PNAISH tem como objetivo facilitar e ampliar o acesso com qualidade da população masculina às ações e aos serviços de assistência integral à saúde da Rede SUS, mediante o enfrentamento racional dos fatores de risco e a atuação nos aspectos socioculturais, sob a perspectiva de gênero, contribuindo de modo efetivo para a redução da morbidade, da mortalidade e a melhoria das condições de saúde ${ }^{10}$. Mais recentemente, pela crescente percepção/aceitação das diferenças entre os gêneros no que tange às demandas e necessidades de saúde, a produção acerca da saúde dos homens vem aumentando de uma forma expressiva ${ }^{12}$.

Neste contexto, a Área Técnica de Saúde do Homem do Ministério da Saúde tem realizado esforços significativos para ampliar a participação deste segmento populacional na ABS, principalmente tornando-o visível aos gestores e trabalhadores da saúde nas potenciais oportunidades de inclusão deste.

Este artigo tem por objetivo descrever as especificidades da atenção à saúde dos homens no âmbito da ESF, conforme a visão do gestor, a demanda dos homens adstritos às unidades avaliadas e as práticas desenvolvidas pelas equipes da ESF (EqSF).

\section{Métodos}

Este artigo é produto do projeto "Fortalecimento e disseminação da Política Nacional de Atenção Integral à Saúde do Homem" que teve como objetivo geral avaliar o impacto da intervenção das equipes de Atenção Básica, em especial da ESF, na implementação das ações de atenção à saúde do homem e identificar as ações e as estratégias utilizadas pela Atenção Básica para fortalecer a PNAISH.

Para este estudo, foram elegíveis os 79 municípios pactuantes da PNAISH em 2009 ou 2010. Destes municípios foram escolhidos apenas aqueles que tinham o Programa Nacional de Melhoria do Acesso e da Qualidade da Atenção Básica (PMAQ-AB) homologado, restando 65. Os municípios foram, então, classificados de acordo com o nível de cobertura do Programa da Saúde da 
Família (PSF): baixa (<30\%), regular (30 a 59\%), boa ( 60 a $79 \%$ ) ou muito boa $\geq 80 \%$ ) e o porte da cidade: pequena $(<100$ mil habitantes), média (100 a 500 mil habitantes) ou grande ( $>500 \mathrm{mil}$ habitantes). Em seguida, foram criados nove estratos, por combinação dessas duas variáveis: baixa cobertura e médio porte (4 municípios); baixa cobertura e grande porte (12 municípios); cobertura regular e médio porte (7 municípios); cobertura regular e grande porte (7 municípios); boa cobertura e médio porte (16 municípios); boa cobertura e grande porte (4 municípios); muito boa cobertura e pequeno porte (3 municípios); muito boa cobertura e médio porte (11 municípios); e muito boa cobertura e grande porte (1 município). Foram selecionados 10 municípios, sendo dois de cada região geográfica brasileira, dos quais ao menos um de cada estrato: Itacoatiara (AM) e Rio Branco (AC) na Região Norte, Aracaju (SE) e Paulista (PE) na Região Nordeste, São Gonçalo (RJ) e Volta Redonda (RJ) na Região Sudeste, Curitiba (PR) e Maringá (PR) na Região Sul, Anápolis (GO) e Campo Grande (MT) na Região Centro-Oeste.

Em cada município selecionado foram sorteadas até $10 \%$ do total de Unidades Básicas de Saúde (UBS), não ultrapassando o máximo de cinco por cidade. Em cada UBS foi sorteada uma equipe da ESF, totalizando 43 equipes: quatro da região Norte, nove da Nordeste e 10 de cada uma das demais regiões (SE, S e CO).

Em cada EqSF foram sorteadas duas famílias da respectiva área de cobertura, para identificação de homens entre 20 e 59 anos de idade, que também foram entrevistados, resultando num total de 10 homens em Anápolis, Aracaju, Campo Grande, Curitiba, Maringá, São Gonçalo e Volta Redonda; oito em Paulista e quatro em Itacoatiara e Rio Branco, num total de 86. Caso a família sorteada não tivesse nenhum morador nesta faixa etária, buscava-se a família de ordem imediatamente superior no cadastro local.

A coleta de dados se deu por meio de formulários específicos, desenvolvidos e pré-testados para este estudo, que foram aplicados por entrevistadores treinados juntos aos gestores das equipes e aos homens sorteados, acompanhados, nestes casos, por um agente comunitário de saúde local. Os formulários continham questões acerca da estrutura da EqSF, do responsável pela equipe, da composição da equipe, da área de cobertura e da rotina de trabalho, além das perguntas específicas sobre a saúde do homem. Foram realizadas análises descritivas dos dados, sendo os resultados apresentados em números absolutos e relativos, ilustrados por meio de gráficos. Os dados foram analisados com auxílio do aplicativo IBM SPSS ${ }^{\circledR}$ versão 20.0.

Neste artigo são descritas as percepções dos gestores a cerca das necessidades de saúde dos homens e as demandas por saúde relatadas pelos homens das áreas das EqSF avaliadas, em particular da motivação para a procura de atendimento e das oportunidades de inclusão dos homens na $\mathrm{AB}$.

Este estudo foi aprovado pelo Comitê de Ética em Pesquisa do Instituto Nacional de Saúde da Mulher, da Criança e do Adolescente Fernandes Figueira da Fundação Oswaldo Cruz, em cumprimento dos preceitos da Resolução 196/96 do Conselho Nacional de Saúde ${ }^{13}$.

\section{Resultados}

A maioria dos gestores era do sexo feminino (37), com graduação em Enfermagem (40). A idade média foi de 39 anos, não havendo diferença entre homens e mulheres. O tempo médio de formação foi de 12 anos, sendo de oito anos o tempo de trabalho na Atenção Básica e de quatro na atual gestão. Todos os gestores tinham 40 horas de contrato, dedicando em média 14 horas para as atividades administrativas.

Sete dos 43 gestores nunca ouviram falar sobre a PNAISH e apenas quatro já participaram de alguma discussão, sendo que a maioria já ouviu falar e 10 já leram algo sobre a PNAISH.

Os homens entrevistados tinham em média 40,5 anos de idade e 8,3 de escolaridade, pertenciam à famílias com média de 3,9 pessoas e 1,3 homens entre 20 a 59 anos, representando $38,1 \%$ da família.

Segundo os gestores das equipes de saúde da família participantes, os três principais motivos que levam os homens à procura de atendimento na ESF se referem à: 1) presença de doença aguda ou crônica, 2) busca de medicamentos, tanto prescrição quanto dispensa e 3) situações específicas da saúde do homem, como disfunção erétil, obstrução urinária, suspeita de câncer de próstata, vasectomia e busca de preservativo. Todavia, a maioria dos homens entrevistados informou ter buscado por atendimento no último ano em razão de: 1) doença aguda (inclui dor repentina), 2) exames de rotina e 3) acidente. Apenas um homem referiu buscar o serviço de saúde para exame da próstata e nenhum para medicamento. Destaca-se a busca por saúde mental, identificada como a quarta causa entre os ho- 
mens, mas pouco considerada pelos gestores ( $\mathrm{Fi}$ gura 1). Diferentemente dos motivos citados para busca e demanda dos homens, os gestores citaram como as três principais queixas apresentadas pelos homens ao procurarem o serviço de saúde: presença de doença $(79,1 \%)$, busca de atestados $(12,4 \%)$ e situações específicas da saúde do homem (7,8\%). Saúde mental surge com menos de $1 \%$, busca de medicamento, exames de rotina e acidentes não apareceram.

Dos 86 homens entrevistados, 77 procuraram por atendimento em serviços de saúde no último ano, sendo 41 em serviços públicos e, destes, 24 na EqSF local. Apenas um homem não foi atendido por falta de profissional no local.

Dos 19 homens que não procuraram por atendimento no último ano, oito não tiveram problema de saúde e 11 tiveram algum problema de saúde, mas sem procura por atendimento principalmente por acharem não ser importante ou necessário (nove de 11 casos); um homem referiu falta de dinheiro para pagamento da consulta e outro medo de médico/injeção. Estes resultados são parcialmente concordantes com a opinião dos gestores que consideraram pouco mais de 10\% de não busca por ausência de doen- ça, $10 \%$ por medo e quase $30 \%$ por valores culturais (o que explica não achar importante ou necessário, “eu não preciso”), mas não concordante com incompatibilidade de horário (quase $30 \%$ ) e espera (cerca de $10 \%$ ).

No que se refere aos dias e horários alternativos de atendimento, apenas uma equipe referiu atender aos sábados das 8 às 13 horas, duas que iniciavam às 6 horas e 11 estendiam o horário até às 19 horas nos dias úteis, totalizando 13 (30,2\%) unidades com horário mais compatível com as disponibilidades de trabalhadores, o que poderia favorecer o aumento do atendimento para os homens. Duas unidades referiram já ter atendido em horário alternativo, mas não observaram redução no número de atendimentos quando passaram a atender apenas no horário comercial em dias úteis. Segundo os homens, o tempo de espera médio para o atendimento no último ano foi de 33 minutos e nenhum deles citou limitação de horário como impedimento para busca de atendimento.

A maioria das EqSF afirmou que convida os homens para consulta pelo menos uma vez ao ano $(90,7 \%)$ e que na presença de sintomas de obstrução urinária ou disfunção erétil há sem-

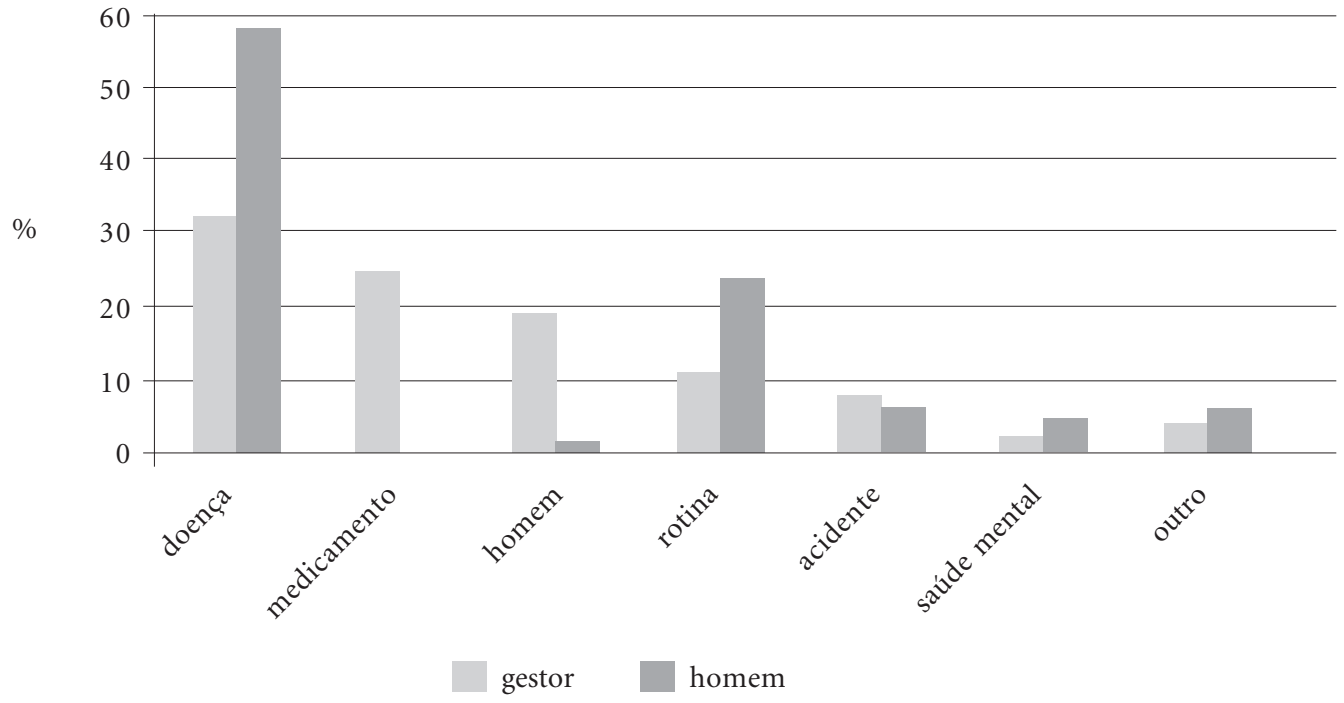

Figura 1. Principais motivos* para procura de atendimento na unidade de saúde da família segundo visão do gestor e demanda dos homens, dezembro de 2012.

* doença = aguda ou crônica; medicamento = prescrição e/ou dispensa; homem = situações específicas da saúde do homem, como disfunção erétil, obstrução urinária, suspeita de câncer de próstata, vasectomia, busca de preservativo; rotina = exames de rotina, check-up; acidente = acidente, curativo; saúde mental = dependência química ou aconselhamento. 
pre encaminhamento para exames diagnósticos (95,3\%). Dentre os homens entrevistados, a maior parte referenciou ter buscado por atendimento em saúde no último ano (77,9\%), mas a minoria respondeu que a EqSF convidou para consulta no último ano $(23,3 \%)$ ou outra atividade $(12,8 \%)$, sendo que as atividades educativas foram as mais frequentes e seis dos 11 homens convidados atenderam ao convite. Dos cinco homens que não participaram dessas atividades, dois alegaram incompatibilidade de horário, um falta de tempo, outro falta de interesse e um falta de acompanhante.

No que se refere às ações de orientação e sensibilização da população masculina quanto às medidas disponíveis para detecção precoce do câncer de próstata em pacientes sintomáticos e disfunção erétil, entre outros agravos do aparelho geniturinário, todas as EqSF referiram fazer o encaminhamento para a realização de exames diagnósticos na presença de sintomas de obstrução urinária ou disfunção erétil, mas apenas $11,6 \%$ tinham alguma atividade específica durante o pré-natal para os pais e nenhuma delas referiu abordar este tema, além da cobertura de atendimento aos homens ter sido identificada em menos de 1\% nas EqSF com alguma informação sobre o número de atendimentos.

As oportunidades para atrair os homens são pouco exploradas pelas EqSF (Figura 2). Das 43 equipes entrevistadas, 21 (48,8\%) referiram realizar algum evento especial para os homens no último ano. Aproximadamente 15\% das EqSF referiram abranger os temas planejamento familiar, consumo abusivo de bebidas alcoólicas e demais drogas, em atividades educativas específicas para o homem. O tema mais comum entre as EqSF foi o tabagismo $(18,6 \%)$ e o menos comum foi a prevenção de violências e acidentes $(9,3 \%)$. Questionadas sobre a estratégia de desenvolvimento destas atividades, a maioria relatou palestras (9), seguidas por grupos educativos (7), consulta individual (3) e distribuição de folhetos (2). Contudo, apenas 7\% do total dos homens entrevistados informaram que a EqSF tinha atividades direcionadas a eles e, dos 80 homens que desconheciam qualquer atividade, 97,5\% gostariam que houvesse alguma.

Quanto à participação oportuna do homem nas atividades de pré-natal, parto, puerpério e no acompanhamento da criança, a maioria das unidades afirmou que pouquíssimos pais participam e que não há atividade específica para os homens nas oito unidades, cuja participação paterna foi reconhecida como quase nenhuma e nenhuma. Nas cinco unidades que referiram ter atividade específica para os homens, a participação nessa fase foi pouco mais de $50 \%$ em uma unidade da região Norte (palestra para conscientização dos cuidados com a saúde), pouco menos de 50\% em uma unidade da região Sudeste (conversando diretamente com o casal) e pouquíssimos em uma unidade do Nordeste (exames HIV e VDRL) e duas do Sul (programa pai presente: exames para HIV e sífilis). Ainda assim, nas oito unidades que não solicitaram a partici-

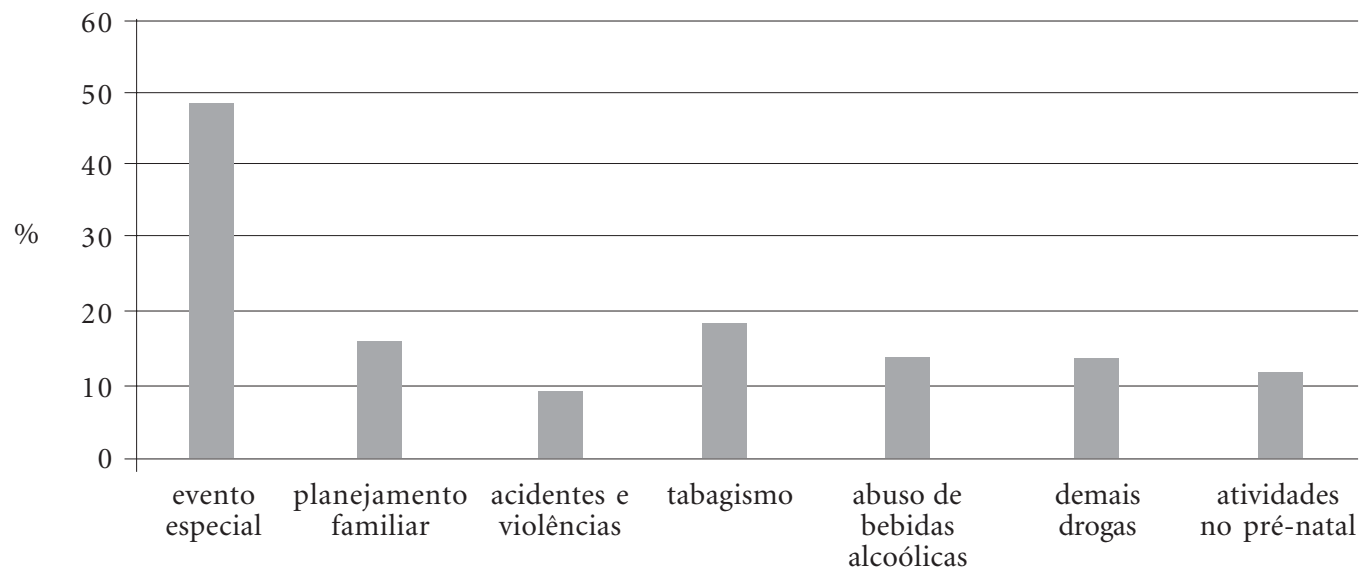

Figura 2 . Oportunidades para participação em atividades específicas para os homens na unidade de saúde da família ,dezembro de 2012. 
pação dos pais nessas atividades, foram obtidas as seguintes participações: nenhuma (uma unidade do SE), quase nenhuma (duas unidades do NE e duas do CO), pouquíssima (uma unidade no $S$ ) e pouco menos da metade (uma unidade no NE e uma no $S$ ).

Das 43 unidades pesquisadas, o homem foi identificado como vítima em apenas 12 delas e como agressor em 31. Na primeira situação, os casos foram identificados principalmente durante o acolhimento e na visita domiciliar, e na segun$\mathrm{da}$, na visita domiciliar. Os encaminhamentos na situação de vítima se referem a cuidados médicos e denúncia, enquanto que na situação de agressor são realizadas denúncia e ações de assistência social, em especial à vítima e não ao agressor. Nesta situação algumas unidades referiram não se envolver, o que realça a dificuldade do setor saúde em trabalhar esta questão.

Do total dos homens entrevistados, $81,6 \%$ já haviam sido atendidos na EqSF da respectiva área de moradia e suas famílias estavam cadastradas na ESF há seis anos. Dos 24 casos atendidos na EqSF, a maioria recebeu prescrição para medicação, sendo que duas pessoas do Nordeste não conseguiram encontrar os medicamentos receitados. Das 16 prescrições, 10 foram dispensadas pelo serviço público sem ônus para o usuário. Quanto aos 11 homens que referiram problema de saúde e não busca de atendimento, quatro se trataram utilizando medicamentos que tinham disponíveis em casa ou com amigos/parentes, três com medicamentos prescritos pela farmácia (um do Centro-Oeste pelo atendente e por telefone) e três com remédio caseiro. A maioria dos homens relatou não haver limitação para falar sobre sua saúde com qualquer profissional da EqSF, independente do sexo. Dos seis casos que referiram algum limite, três usuários preferiam ser atendidos por profissional do sexo masculino (1 no NE e 2 no CO), dois referiram timidez (SE e CO) e um gostaria de maior aproximação da equipe a fim oportunizar abordagem sobre problemas familiares (CO).

\section{Discussão}

Dados da Pesquisa Nacional por Amostra de Domicílios $(\mathrm{PNAD} / 2008)^{14}$ apontaram que as principais razões, referidas pela população total, para a busca de atendimento médico foram: doenças (50\%), seguidas por puericultura, vacinação ou outros atendimentos de prevenção (22\%), problemas odontológicos (14\%) e acidentes e le- são (6\%). Embora, em ambos os sexos, as doenças tenham sido a principal responsável pela procura por atendimento em saúde, as mulheres referiram maior busca por serviços de vacinação ou prevenção ( $24 \%$ e $19 \%$ entre o sexo masculino), enquanto que entre os homens, os motivos mais frequentes para a busca de atendimento em saúde foram os acidentes e as violências ( $8,9 \%$ e 4,1\% entre as mulheres $)^{14}$. A doença, enquanto primeira causa de procura também foi identificada neste estudo, tanto nas citações dos gestores como dos próprios homens. Positivamente, a segunda causa relatada pelos homens foram exames de rotina, reforçando um maior cuidado com sua própria saúde, o que pode ser o principio da transição de um cuidado curativo para a prevenção.

Neste estudo, a grande maioria dos entrevistados buscou serviços de saúde do Sistema Único de Saúde (SUS), o que alude o estado de saúde e as condições de acesso aos serviços, uma vez que o ato de procurar um serviço de saúde depende, principalmente, de fatores relacionados aos serviços disponíveis e de fatores próprios de cada indivíduo. Capilheira e Santos ${ }^{15}$ e Fernandes et al. ${ }^{16}$ afirmam que há uma maior utilização dos serviços por indivíduos que percebem sua saúde como regular ou ruim, corroborando com os achados encontrados em estudo qualitativo realizado em quatro estados brasileiros ${ }^{4}$. Leal et al. ${ }^{17}$ justificam que um problema reconhecido é a rede de apoio deficitária, destacada, nesse cenário, pela ausência, em função da PNAISH, de fluxos específicos para homens, o que vai de encontro às expectativas masculinas de rapidez e resolubilidade. Os profissionais de saúde acreditam que o longo tempo de espera em atendimentos também agrava o problema do absenteísmo masculino.

Do total de homens entrevistados, $58 \%$ daqueles que não procuraram por atendimento tiveram problemas de saúde, que os levaram à busca de tratamento sem orientação clínica, reforçando a cultura da automedicação. Estudo realizado em um município de São Paulo apontou que $91,1 \%$ dos domicílios apresentavam estoques de medicamentos e a aquisição destes ocorreu principalmente em farmácias e drogarias, uma vez que os medicamentos adquiridos nas ESF ou outras unidades de saúde requerem prescrição médica, corroborando os dados encon$\operatorname{trados}^{18}$. Homens, diante de algum problema de saúde, procuram medidas de tratamento alternativas, desde o uso de chás até automedicação, buscando orientação de algum farmacêutico, quando possível ${ }^{7}$. Pinheiro et al. ${ }^{19}$ apontaram a maior incidência masculina na procura de servi- 
ços emergenciais, tais como farmácias, o que sugere que os homens preferem respostas mais objetivas às suas demandas, considerando que nesses espaços os homens seriam atendidos mais rapidamente e conseguiriam expor seus problemas com maior facilidade $e^{6,13}$.

Outro fator que sugere a não procura por serviços de saúde, por parte dos homens, é que muitos locais de trabalho só abonam a falta mediante atestado médico, que não é fornecido pelos serviços de saúde no caso de simples marcação de consulta, participação de grupos, busca de medicamentos e outras atividades vinculadas à prevenção ${ }^{3}$.

Um fator a ser considerado quanto à inserção do homem na atenção básica refere-se à precarização dos serviços públicos em relação ao atendimento. Pesquisa qualitativa realizada na cidade do Rio de Janeiro, junto a dois grupos: Idez homens com idade entre 45 e 57 anos, com baixa ou nenhuma escolaridade e, II- oito homens com idade entre 40 e 64 anos, com ensino superior, demonstrou que o grupo I, supostamente por ter o menor poder aquisitivo quando comparados ao grupo II, tem menor preocupação com as questões relativas aos cuidados de saúde, dedicando maior atenção para o trabalho e o sustento da casa e da família. Tal comportamento reflete na busca pelos serviços de saúde, já que os homens desse grupo referiram dificuldade de acesso aos serviços assistenciais, relataram que enfrentam filas para conseguir consultas e que suas demandas, possivelmente, não seriam resolvidas no mesmo dia, o que prejudicaria seu desempenho no trabalho ${ }^{7}$.

Alguns estudos têm relatado a invisibilidade dos homens na atenção primária à saúde ${ }^{20,21}$, uma vez que estes serviços, historicamente, têm desenvolvido mais ações destinadas à saúde de mulheres, crianças e idosos ${ }^{6,22}$. A ausência dos homens nas UBS pode ser explicada em virtude destas não disponibilizarem atividades ou programas direcionados especificamente para este público e os homens preferirem utilizar serviços que respondem mais rapidamente e objetivamente às suas demandas, como farmácia e pronto socorro ${ }^{6}$. Durante as ações de promoção da saúde e divulgação da PNAISH desenvolvidas na primeira semana estadual de atenção à saúde do homem, realizada para funcionários e alunos da Faculdade Federal da Paraíba, no município de João Pessoa, observou-se que muitos homens demonstraram surpresa com a existência de uma política específica para a sua saúde e satisfação pela possibilidade de sua inclusão nos serviços de atenção primária à saúde ${ }^{9}$. A vivência dessas ações revelou ainda que os participantes apresentaram pouco conhecimento em relação à promoção da saúde e à prevenção de agravos ${ }^{9}$.

No estudo qualitativo realizado junto a 11 homens com idade entre 25 e 56 anos, adstritos em uma área assistida por uma EqSF, na cidade de Parnamirim no estado do Rio Grande do Norte, as autoras ${ }^{23}$ constataram a necessidade de reestruturação das UBS, sugerindo estender o horário de atendimento para o período noturno, o que facilitaria a inserção tanto dos homens que estão no mercado formal de trabalho, quanto dos trabalhadores autônomos que muitas vezes obtém renda pela quantidade de horas trabalhadas. Os entrevistados demonstraram desconhecimento quanto às funções da EqSF e predominaram as opiniões negativas sobre essa estratégia, principalmente no que se refere às dificuldades para agendamento de consultas médicas e exames.

Outros estudos, ainda, ao avaliarem casos, em municípios das cinco macrorregiões do país, apontaram que não há, de fato, ações continuadas voltadas à população masculina na faixa etária de 20 a 59 anos de idade, o que embasa os dados observados neste estudo, no qual a minoria dos homens respondeu ter recebido convite para consultas, ou atividades de saúde ${ }^{6,17}$. As atividades existentes e direcionadas aos homens são geralmente voltadas para ações assistenciais ou atividades pontuais, como a realização da "Semana do Homem", não contemplando, assim, as diretrizes propostas na PNAISH ${ }^{6,17}$.

Estudo qualitativo ${ }^{24}$, realizado junto a nove homens com idade entre 19 e 62 anos e com baixo nível de escolaridade, vai ao encontro dos dados deste estudo, quanto à participação masculina no pré-natal de suas parceiras, na área de abrangência da ESF do município de Catu, no estado da Bahia. O estudo demonstrou que a maioria das gestações não foi planejada e que os homens não acompanhavam as consultas do pré-natal. A maioria dos entrevistados relatou que tinha ciência que suas parceiras realizavam o pré-natal, entretanto, não soube explicar o que é esse tipo de assistência; do total de entrevistados, apenas um acompanhou a parceira na consulta, cabe ressaltar que nesse caso a companheira tinha deficiência auditiva e na fala. Dentre os principais motivos para a não participação das consultas de pré-natal, os homens destacaram a falta de tempo, em virtude do trabalho, e desinteresse em participar deste tipo de atendimento.

Apesar da tímida implantação da PNAISH, o público dos serviços de saúde permanece majo- 
ritariamente feminino e infantil. Gomes et al. ${ }^{7}$, em estudo sobre a percepção dos homens a respeito dos serviços de atenção primária a saúde, mostraram que os mesmos se destinam às pessoas idosas, às mulheres e às crianças, e são considerados pelos homens como um espaço "feminilizado", o que se espera estar em modificação ao longo dos últimos anos, uma vez que o presente estudo aponta quase a totalidade dos homens com interesse em atividades da ESF.

A Promoção da Saúde e a prevenção de agravos têm sido focalizadas como eixos fundamentais na $\mathrm{ESF}^{11}$. Os homens estão mais vulneráveis a problemas de saúde, em virtude da sua baixa acessibilidade aos serviços de atenção primária9. Diante disto, a PNAISH orientou a formulação de diretrizes e ações voltadas fundamentalmente para a atenção integral, com vistas à prevenção e à promoção da saúde, à qualidade de vida e à educação, como dispositivos estratégicos de incentivo às mudanças comportamentais ${ }^{10}$.

A principal causa de óbito entre os homens das cidades avaliadas foram, em 2010, as causas externas $(36,4 \%)$, seguidas por doenças do aparelho circulatório $(18,6 \%)$ e neoplasias $(11,1 \%)^{25}$. Quanto às principais morbidades - causas de internação, observou-se o predomínio de causas externas $(15,7 \%)$, doenças do aparelho respiratório $(15,0 \%)$ e doenças do aparelho circulatório $(12,7 \%)$. As doenças do aparelho geniturinário representaram $6,1 \%$ das causas de internação em $2010^{25}$. Embora os gestores considerem acidentes como a quinta principal causa para a procura da UBS pelo homem, as causas externas (acidentes e violências) aparecem como a primeira causa de óbitos em todos os municípios e como primeira causa de internação na maioria deles.

As causas externas - violências e acidentes exercem grande impacto na qualidade de vida e nas condições de saúde da população, pois atingem um número muito maior de pessoas do que aquelas que se encontram diretamente envolvidas e seus efeitos ultrapassam o sofrimento individual e coletivo, incidindo na cultura e no modo de viver das pessoas ${ }^{26}$. No Brasil, em 2010, foram registrados no Sistema de Informação sobre Mortalidade (SIM) 75 óbitos por 100.000 habitantes (126,5/100 mil em homens e 25,6/100 mil em mulheres) ${ }^{25}$. A notificação de todo caso suspeito ou confirmado de violência doméstica, sexual e/ou outras contra homens e mulheres atendidos em serviços de saúde passou a ser compulsória em todo o território nacional em $2011^{27}$.

As visitas domiciliares são importante fonte de informação para a identificação de situações de violência envolvendo homens, tanto como vítima como agressor, porém o acolhimento se dá basicamente para a vítima e o encaminhamento se refere principalmente aos cuidados de saúde e denúncia para a vítima, reafirmando a necessidade da validação e da utilização de protocolos clínicos e diretrizes terapêuticas para homens e mulheres em situação de violência na rotina de trabalho das equipes da ESF/UBS a partir da complexidade e importância deste tema na área da saúde.

Em relação aos agravos específicos dos homens, o câncer de próstata é um dos principais problemas relacionados ao aparelho geniturinário e corresponde à segunda causa mais comum de óbito por câncer no Brasil, atingindo principalmente homens com 50 ou mais anos de idade.

Embora a adoção de um programa exclusivo de rastreamento populacional para esta patologia torne-se inviável, pois com o aumento da idade ocorre uma maior frequência de tumores latentes - que na maioria das vezes evoluirão de maneira lenta e não nociva à saúde, além de uma alta morbimortalidade relacionada aos atuais procedimentos terapêuticos empregados ${ }^{28}$, fazse de suma importância a realização do rastreamento oportunístico e da vigilância ativa naqueles homens que possuem histórico familiar da doença em parentes de $1^{\circ}$ grau e/ou com o recorte de raça/cor presente, a partir dos 40 anos, a fim de que uma morbimortalidade precoce e desnecessária possa ser prevenida e combatida.

Estes dados também corroboram com os observados na pesquisa que avaliou as ações iniciais da implantação da PNAISH, realizada junto a gestores das 27 unidades federadas e de 26 municípios que iniciaram a implantação da política, em que há pouca ou nenhuma familiaridade dos entrevistados com o tema ${ }^{29}$. Leal et al. ${ }^{17}$, em estudo que buscou compreender o ponto de vista dos profissionais do SUS acerca da PNAISH em cinco municípios do Brasil, apontaram que há uma grande diferença entre os municípios sobre os conhecimentos das diretrizes e rotinas contidas na política, por parte dos gerentes e dos profissionais da assistência.

Estudos sobre a utilização de serviços de saúde pela população contribuem para o aprimoramento da assistência, uma vez que permitem identificar níveis de cobertura e grupos populacionais excluídos, auxiliando no planejamento em saúde $^{30}$.

As EqSF têm como estratégia abordar o processo saúde/doença no contexto familiar e ambiental, tendo como ação básica a prevenção dos 
agravos mais frequentes à saúde, além da promoção da saúde de modo a possibilitar o acesso universal e contínuo a serviços de saúde de qualidade, conforme os princípios de universalização, equidade, descentralização, integralidade e participação da comunidade - mediante o cadastramento e a vinculação dos usuários ${ }^{27}$. No que se refere à Saúde do Homem, ainda há muito a ser feito: desde adequação da estrutura para atendimento na atenção básica (São Gonçalo, por exemplo); motivação e desenvolvimento de ações de promoção contra os agravos mais frequentes nesta população (causas externas, por exemplo) de modo a corresponsabilizar o homem pela sua própria saúde e, consequentemente, do meio do qual participa, colaborando para que ele aos poucos possa ir se percebendo como um sujeito de cuidados e de direito à saúde.

Ainda que este estudo não traga a representação de todo o público masculino, pôde-se con- cluir que, embora haja interesse dos homens em ampliar sua participação nas atividades desenvolvidas pelas equipes das ESF/UBS, uma vez que dirigidas às suas reais necessidades, ainda há lacunas significativas a serem preenchidas, desde a adequação da estrutura/ambiência para o atendimento na atenção básica à motivação e o desenvolvimento de ações de promoção, tratamento e recuperação dos agravos mais frequentes nesta população (causas externas, por exemplo).

Desta maneira, os homens podem estar cada vez mais incluídos e integrados na lógica sistêmica das populações atendidas e dos serviços ofertados pela rede SUS, contribuindo assim para a desconstrução do paradigma cultural da masculinidade vigente e do imaginário simbólico coletivo que reforça a invulnerabilidade masculina, semeando uma melhor qualidade de vida e saúde para todos(as).

\section{Colaboradores}

EC Moura, W Santos, ACM Neves, R Gomes e E Schwarz participaram igualmente de todas as etapas de elaboração do artigo.

\section{Referências}

1. Scott J. Gênero: uma categoria útil para a análise histórica. Educação \& Realidade 1995; 20(2):71-99.

2. Machin R, Couto MT, Silva GSN, Schraiber LB, Gomes R, Figueiredo WS, Valença AO, Pinheiro TF. Concepções de gênero, masculinidade e cuidados em saúde: estudo com profissionais de saúde da atenção primária. Cien Saude Colet 2011; 16(11): 4503-4512.

3. Knauth DR, Couto MT, Figueiredo WS. A visão dos profissionais sobre a presença e as demandas dos homens nos serviços de saúde: perspectivas para a análise da implantação da Política Nacional de Atenção Integral à Saúde do Homem. Cien Saude Colet 2012; 17(10):2617-2626.

4. Schraiber LB, Figueiredo WS, Gomes R, Couto MT, Pinheiro TF, Machin R, Silva GSN, Valença O. Necessidades de saúde e masculinidades: atenção primária no cuidado aos homens. Cad Saude Publica 2010; 26(5):961-970.

5. Moura EC, Neves ACM, Sá NNB, Silva AS, Santos W. Perfil da situação de saúde do homem no Brasil. Rio de Janeiro: Fiocruz; 2012. (Relatório técnico)

6. Figueiredo W. Assistência à saúde dos homens: um desafio para os serviços de atenção primária. Cien Saude Colet 2005; 10(1):105-109.

7. Gomes R, Nascimento EF, Araújo FC. Porque os homens buscam menos os serviços de saúde do que as mulheres? As explicações de homens com baixa escolaridade e homens com ensino superior. Cad Saude Publica 2007; 23(3):565-574.

8. Valdés T, Olavarría J. Masculinidades y equidad de género en América Latina. Santiago: Flacso; 1998. 
9. Fontes WD, Barboza TM, Leite MC, Fonseca RLS, Santos LCF, Nery TCL. Atenção à saúde do homem: interlocução entre ensino e serviço. Acta Paul Enferm 2011; 24(3):430-433.

10. Brasil. Ministério da Saúde (MS). Secretaria de Atenção à Saúde. Departamento de Ações Programáticas e Estratégicas. Política Nacional de Atenção Integral à Saúde do Homem: princípios e diretrizes. Brasília: MS; 2009.

11. Rodrigues MP, Lima KC, Roncalli AG. A representação social do cuidado no programa saúde da família na cidade de Natal. Cien Saude Colet 2008; 13(1):71-82.

12. Couto MT, Gomes R. Homens, saúde e políticas públicas: a equidade de gênero em questão. Cien Saude Colet 2012; 17(1):2569-2578.

13. Brasil. Ministério da Saúde (MS). Conselho Nacional de Saúde. Resolução no 196 de 10 de outubro de 1996. Diretrizes e Normas Regulamentadoras de Pesquisas Envolvendo Seres Humanos. Diário Oficial da União 1996; 16 out.

14. Instituto Brasileiro de Geografia e Estatística (IBGE). Pesquisa Nacional por Amostra de Domicílios: um panorama da saúde no Brasil - acesso e utilização dos serviços, condições de saúde e fatores de risco e proteção à saúde 2008. Rio de Janeiro: IBGE; 2010. (Relatório técnico)

15. Capilheira MF, Santos IS. Fatores individuais associados à utilização de consultas médicas por adultos. Rev Saude Publica 2006;40(3):436-443.

16. Fernandes LCL, Bertoldi AD, Barros AJD. Health service use in a population covered by the Estratégia de Saúde da Família (Family Health Strategy). Rev Saude Publica 2009; 43(4):595-603.

17. Leal AF, Figueiredo WS, Nogueira-da-Silva GS. O percurso da Política Nacional de Atenção Integral à Saúde dos Homens (PNAISH), desde a sua formulação até sua implementação nos serviços públicos locais de atenção à saúde. Cien Saude Colet 2012; 17(10):2607-2616.

18. Mastroianni PC, Lucchetta RC, Sarra JR, Galduroz JCF. Estoque doméstico e uso de medicamentos em uma população cadastrada na estratégia saúde da família no Brasil. Rev Panam Salud Publica 2011; 29(5):358-364.

19. Pinheiro RS, Viacava F, Travassos C, Brito AS. Gênero, morbidade, acesso e utilização de serviços de saúde no Brasil. Cien Saude Colet 2002; 7(4):687-707.

20. Couto MT, Pinheiro TF, Valença O, Machin R, Silva GSN, Gomes R, Schraiber LB, Figueiredo WS. O homem na atenção primária à saúde: discutindo (in)visibilidade a partir da perspectiva de gênero. Interface (Botucatu) 2010; 14(33):257-270.

21. Gomes R, Moreira MCN, Nascimento EF, Rebello LEFS, Couto MT, Schraiber LB. Os homens não vêm! Ausência e/ou invisibilidade masculina na atenção primária. Cien Saude Colet 2011;16(Supl. 1):S983-S992.
22. Andrade LOM, Barreto ICHC, Bezerra RC. Atenção primária a saúde e estratégia saúde da família. In: Campos GWS, Minayo MCS, Akerman M, Drumond Júnior M, Carvalho YM, editores. Tratado de Saúde Coletiva. São Paulo, Rio de Janeiro: Hucitec, Fiocruz; 2008. p. 783-836.

23. Brito RS, Santos DLA, Maciel PSO. Olhar masculino acerca do atendimento na Estratégia Saúde da Família. Rev Rene 2010; 41(4):135-142.

24. Oliva TA, Nascimento ER, Santo FRE. Percepções e experiências de homens relativas ao pré-natal e parto de suas parceiras. Rev enferm. 2010; 18(3):435-40.

25. Brasil. Ministério da Saúde (MS). Secretaria de Vigilância em Saúde. Departamento de Análise de Situação de Saúde. Mortalidade por acidentes e violências no Brasil: situação em 2010 e tendências de 2011 a 2010. In: Brasil. Saúde Brasil 2011: uma análise da situação de saúde e a vigilância da saúde da mulher. Brasília: MS; 2012. p. 249-278.

26. World Health Organization (WHO). World report on violence and health [relatório técnico online]. Geneva: WHO; 2002. [acessado $2013 \mathrm{fev} 28$ ]. Disponível em: http://whqlibdoc.who.int/publications/ 2002/9241545615_eng.pdf.

27. Brasil. Ministério da Saúde (MS). Secretaria de Vigilância em Saúde. Departamento de Análise de Situação de Saúde. Viva: instrutivo de notificação de violência doméstica, sexual e outras violências [relatório técnico]. Brasília: MS; 2011.

28. Brasil. Ministério da Saúde (MS). Secretaria de Atenção à Saúde. Departamento de Atenção Básica. Cadernos de Atenção Básica: rastreamento. Brasília: MS; 2010.

29. Gomes R, Leal AF, Knauth D, Silva GSN. Sentidos atribuídos à política voltada para a Saúde do Homem. Cien Saude Colet 2012;17(10):2589-2596.

30. Dias-da-Costa JS, Presser AD, Zanolla AF, Ferreira DG, Perozzo G, Freitas IBA, Portolan LT, Tavares RT, Olinto MTA, Pattussi MP. Utilização dos serviços ambulatoriais de saúde por mulheres: estudo de base populacional no sul do Brasil. Cad Saude Publica 2008; 24(12):2843-2851.

Artigo apresentado em 02/05/2013

Aprovado em 23/05/2013

Versão final apresentada em 24/05/2013 